\title{
A global simulation of brown carbon: implications for photochemistry and direct radiative effect
}

Duseong S. Jo et al.

Correspondence to: Rokjin J. Park (rjpark@snu.ac.kr)

The copyright of individual parts of the supplement might differ from the CC-BY 3.0 licence. 
Table S1. BC emission ratios between 2007 and 2000 for each region and month. Regions used in this table are defined by the ACCMIP inventory.

\begin{tabular}{cccccccccccccc}
\hline Region & Annual & Jan & Feb & Mar & Apr & May & Jun & Jul & Aug & Sep & Oct & Nov & Dec \\
\hline Canada & 0.60 & 1.04 & 0.86 & 0.79 & 0.59 & 0.43 & 0.24 & 0.12 & 0.24 & 0.41 & 0.64 & 0.83 & 1.01 \\
USA & 0.56 & 0.97 & 0.80 & 0.74 & 0.55 & 0.40 & 0.22 & 0.11 & 0.23 & 0.39 & 0.60 & 0.78 & 0.94 \\
Mexico & 0.95 & 1.63 & 1.35 & 1.25 & 0.94 & 0.69 & 0.39 & 0.22 & 0.40 & 0.66 & 1.02 & 1.30 & 1.58 \\
Rest_Central_America & 1.02 & 1.61 & 1.35 & 1.28 & 1.01 & 0.79 & 0.52 & 0.36 & 0.52 & 0.76 & 1.08 & 1.33 & 1.58 \\
Brazil & 1.01 & 0.39 & 0.51 & 0.79 & 1.04 & 1.36 & 1.50 & 1.57 & 1.41 & 1.22 & 1.04 & 0.78 & 0.57 \\
Venuzuela & 1.02 & 1.38 & 1.19 & 1.19 & 1.01 & 0.90 & 0.71 & 0.59 & 0.69 & 0.85 & 1.07 & 1.23 & 1.39 \\
Argentina & 1.01 & 0.21 & 0.38 & 0.72 & 1.05 & 1.44 & 1.64 & 1.75 & 1.54 & 1.29 & 1.03 & 0.70 & 0.41 \\
Rest_South_America & 1.01 & 0.61 & 0.66 & 0.88 & 1.03 & 1.26 & 1.32 & 1.35 & 1.25 & 1.14 & 1.04 & 0.88 & 0.75 \\
Nothren_Africa & 1.09 & 1.89 & 1.56 & 1.45 & 1.08 & 0.78 & 0.43 & 0.22 & 0.44 & 0.75 & 1.17 & 1.51 & 1.84 \\
Western_Africa & 1.39 & 1.64 & 1.46 & 1.53 & 1.39 & 1.34 & 1.16 & 1.04 & 1.12 & 1.25 & 1.46 & 1.57 & 1.70 \\
Eastern_Africa & 1.16 & 1.27 & 1.15 & 1.24 & 1.16 & 1.16 & 1.04 & 0.96 & 1.00 & 1.08 & 1.21 & 1.27 & 1.33 \\
Rest_Southern_Africa & 1.21 & 0.61 & 0.71 & 1.00 & 1.23 & 1.55 & 1.67 & 1.73 & 1.58 & 1.40 & 1.24 & 0.99 & 0.79 \\
South_Africa & 2.04 & 0.41 & 0.77 & 1.45 & 2.10 & 2.90 & 3.30 & 3.52 & 3.10 & 2.60 & 2.07 & 1.40 & 0.83 \\
France & 0.87 & 1.50 & 1.24 & 1.15 & 0.85 & 0.62 & 0.34 & 0.18 & 0.35 & 0.60 & 0.93 & 1.20 & 1.46 \\
Germany & 0.87 & 1.51 & 1.24 & 1.15 & 0.86 & 0.62 & 0.34 & 0.18 & 0.35 & 0.60 & 0.93 & 1.20 & 1.46 \\
Italy & 0.86 & 1.48 & 1.22 & 1.13 & 0.84 & 0.61 & 0.34 & 0.17 & 0.35 & 0.59 & 0.91 & 1.18 & 1.44 \\
United_Kingdom & 0.85 & 1.48 & 1.22 & 1.13 & 0.84 & 0.61 & 0.34 & 0.17 & 0.35 & 0.59 & 0.91 & 1.18 & 1.43 \\
Rest_Western_Europe & 0.89 & 1.54 & 1.27 & 1.18 & 0.88 & 0.63 & 0.35 & 0.18 & 0.36 & 0.61 & 0.95 & 1.23 & 1.49 \\
Rest_Central_Europe & 0.97 & 1.67 & 1.38 & 1.28 & 0.95 & 0.69 & 0.38 & 0.20 & 0.39 & 0.67 & 1.03 & 1.33 & 1.62
\end{tabular}




\begin{tabular}{|c|c|c|c|c|c|c|c|c|c|c|c|c|c|}
\hline Baltic_States & 0.72 & 1.24 & 1.02 & 0.95 & 0.71 & 0.51 & 0.28 & 0.15 & 0.29 & 0.49 & 0.77 & 0.99 & 1.21 \\
\hline Turkey & 0.85 & 1.47 & 1.21 & 1.12 & 0.84 & 0.60 & 0.33 & 0.17 & 0.35 & 0.59 & 0.91 & 1.17 & 1.43 \\
\hline Ukraine & 0.60 & 1.04 & 0.86 & 0.80 & 0.59 & 0.43 & 0.24 & 0.12 & 0.25 & 0.42 & 0.64 & 0.83 & 1.01 \\
\hline Asia-Stan & 0.64 & 1.11 & 0.92 & 0.85 & 0.63 & 0.46 & 0.25 & 0.13 & 0.26 & 0.44 & 0.69 & 0.89 & 1.08 \\
\hline Russia & 0.69 & 1.20 & 0.99 & 0.92 & 0.68 & 0.49 & 0.27 & 0.14 & 0.28 & 0.48 & 0.74 & 0.96 & 1.16 \\
\hline Middle_East & 3.41 & 5.87 & 4.85 & 4.50 & 3.36 & 2.44 & 1.36 & 0.72 & 1.41 & 2.36 & 3.64 & 4.69 & 5.70 \\
\hline zIndia & 1.10 & 1.85 & 1.53 & 1.43 & 1.09 & 0.81 & 0.48 & 0.28 & 0.49 & 0.78 & 1.17 & 1.49 & 1.80 \\
\hline Rest_South_Asia & 1.11 & 1.87 & 1.55 & 1.45 & 1.09 & 0.81 & 0.48 & 0.28 & 0.49 & 0.78 & 1.18 & 1.51 & 1.82 \\
\hline South_Korea & 0.97 & 1.67 & 1.38 & 1.28 & 0.95 & 0.69 & 0.38 & 0.20 & 0.39 & 0.67 & 1.03 & 1.33 & 1.62 \\
\hline North_Korea & 1.06 & 1.84 & 1.52 & 1.41 & 1.05 & 0.76 & 0.42 & 0.22 & 0.43 & 0.73 & 1.14 & 1.47 & 1.79 \\
\hline China & 1.12 & 1.94 & 1.60 & 1.48 & 1.10 & 0.80 & 0.44 & 0.23 & 0.46 & 0.77 & 1.20 & 1.55 & 1.88 \\
\hline Taiwan & 1.22 & 2.10 & 1.74 & 1.61 & 1.20 & 0.87 & 0.48 & 0.25 & 0.49 & 0.84 & 1.30 & 1.68 & 2.04 \\
\hline Thailand & 1.07 & 1.64 & 1.38 & 1.33 & 1.06 & 0.86 & 0.59 & 0.42 & 0.59 & 0.82 & 1.13 & 1.38 & 1.62 \\
\hline Rest_Southeastern_Asia & 1.03 & 1.60 & 1.35 & 1.29 & 1.02 & 0.82 & 0.56 & 0.40 & 0.56 & 0.79 & 1.10 & 1.34 & 1.58 \\
\hline Indonesia & 0.97 & 0.75 & 0.75 & 0.91 & 0.98 & 1.12 & 1.12 & 1.12 & 1.07 & 1.02 & 1.00 & 0.91 & 0.85 \\
\hline Japan & 0.54 & 0.93 & 0.76 & 0.71 & 0.53 & 0.38 & 0.21 & 0.11 & 0.22 & 0.37 & 0.57 & 0.74 & 0.90 \\
\hline Australia & 0.71 & 0.15 & 0.27 & 0.51 & 0.74 & 1.01 & 1.15 & 1.23 & 1.09 & 0.91 & 0.73 & 0.49 & 0.29 \\
\hline New_Zealand & 0.67 & 0.14 & 0.26 & 0.48 & 0.70 & 0.96 & 1.09 & 1.16 & 1.03 & 0.86 & 0.68 & 0.46 & 0.27 \\
\hline Rest_Oceania & 1.06 & 0.40 & 0.53 & 0.83 & 1.09 & 1.43 & 1.58 & 1.66 & 1.49 & 1.29 & 1.09 & 0.81 & 0.58 \\
\hline
\end{tabular}


Table S2. Same as Table S1 but for OC emission ratios.

\begin{tabular}{|c|c|c|c|c|c|c|c|c|c|c|c|c|c|}
\hline Region & Annual & Jan & Feb & Mar & Apr & May & Jun & Jul & Aug & Sep & Oct & Nov & Dec \\
\hline Canada & 0.86 & 1.48 & 1.22 & 1.13 & 0.84 & 0.61 & 0.34 & 0.17 & 0.35 & 0.59 & 0.91 & 1.18 & 1.44 \\
\hline USA & 0.82 & 1.41 & 1.16 & 1.08 & 0.80 & 0.58 & 0.32 & 0.17 & 0.33 & 0.56 & 0.87 & 1.12 & 1.37 \\
\hline Mexico & 1.14 & 1.94 & 1.61 & 1.49 & 1.12 & 0.82 & 0.47 & 0.26 & 0.48 & 0.79 & 1.21 & 1.56 & 1.89 \\
\hline Rest_Central_America & 1.05 & 1.63 & 1.37 & 1.31 & 1.04 & 0.84 & 0.57 & 0.40 & 0.57 & 0.80 & 1.11 & 1.36 & 1.60 \\
\hline Brazil & 1.12 & 0.43 & 0.56 & 0.87 & 1.15 & 1.50 & 1.66 & 1.74 & 1.56 & 1.35 & 1.14 & 0.86 & 0.62 \\
\hline Venuzuela & 1.63 & 2.21 & 1.91 & 1.91 & 1.63 & 1.45 & 1.14 & 0.95 & 1.11 & 1.36 & 1.72 & 1.97 & 2.22 \\
\hline Argentina & 1.20 & 0.24 & 0.46 & 0.86 & 1.24 & 1.71 & 1.95 & 2.08 & 1.83 & 1.54 & 1.22 & 0.83 & 0.49 \\
\hline Rest_South_America & 1.06 & 0.64 & 0.69 & 0.92 & 1.08 & 1.31 & 1.37 & 1.40 & 1.30 & 1.19 & 1.09 & 0.92 & 0.78 \\
\hline Nothren_Africa & 1.09 & 1.89 & 1.56 & 1.45 & 1.08 & 0.78 & 0.43 & 0.22 & 0.45 & 0.75 & 1.17 & 1.51 & 1.84 \\
\hline Western_Africa & 1.28 & 1.51 & 1.34 & 1.41 & 1.29 & 1.25 & 1.08 & 0.97 & 1.04 & 1.16 & 1.35 & 1.45 & 1.56 \\
\hline Eastern_Africa & 1.10 & 1.23 & 1.11 & 1.19 & 1.11 & 1.10 & 0.98 & 0.91 & 0.95 & 1.02 & 1.15 & 1.21 & 1.28 \\
\hline Rest_Southern_Africa & 1.15 & 0.58 & 0.68 & 0.96 & 1.18 & 1.47 & 1.58 & 1.64 & 1.50 & 1.33 & 1.18 & 0.95 & 0.76 \\
\hline South_Africa & 2.38 & 0.49 & 0.90 & 1.69 & 2.46 & 3.39 & 3.86 & 4.12 & 3.63 & 3.04 & 2.42 & 1.64 & 0.97 \\
\hline France & 0.76 & 1.31 & 1.08 & 1.00 & 0.75 & 0.54 & 0.30 & 0.15 & 0.31 & 0.52 & 0.81 & 1.04 & 1.27 \\
\hline Germany & 0.75 & 1.30 & 1.07 & 0.99 & 0.74 & 0.54 & 0.30 & 0.15 & 0.31 & 0.52 & 0.80 & 1.04 & 1.26 \\
\hline Italy & 0.75 & 1.29 & 1.07 & 0.99 & 0.73 & 0.53 & 0.29 & 0.15 & 0.30 & 0.51 & 0.80 & 1.03 & 1.25 \\
\hline United_Kingdom & 0.73 & 1.26 & 1.04 & 0.96 & 0.72 & 0.52 & 0.29 & 0.15 & 0.30 & 0.50 & 0.78 & 1.00 & 1.22 \\
\hline Rest_Western_Europe & 0.80 & 1.39 & 1.15 & 1.06 & 0.79 & 0.57 & 0.32 & 0.16 & 0.33 & 0.55 & 0.86 & 1.11 & 1.35 \\
\hline Rest_Central_Europe & 0.83 & 1.43 & 1.18 & 1.10 & 0.82 & 0.59 & 0.33 & 0.17 & 0.34 & 0.57 & 0.89 & 1.14 & 1.39 \\
\hline Baltic_States & 0.81 & 1.39 & 1.15 & 1.07 & 0.79 & 0.57 & 0.32 & 0.16 & 0.33 & 0.56 & 0.86 & 1.11 & 1.35 \\
\hline
\end{tabular}




\begin{tabular}{crrrrrrrrrrrrr} 
Turkey & 0.79 & 1.37 & 1.13 & 1.05 & 0.78 & 0.56 & 0.31 & 0.16 & 0.32 & 0.55 & 0.85 & 1.09 & 1.33 \\
Ukraine & 0.75 & 1.30 & 1.07 & 0.99 & 0.74 & 0.53 & 0.29 & 0.15 & 0.30 & 0.52 & 0.80 & 1.03 & 1.26 \\
Asia-Stan & 0.76 & 1.32 & 1.09 & 1.01 & 0.75 & 0.54 & 0.30 & 0.16 & 0.31 & 0.52 & 0.81 & 1.05 & 1.28 \\
Russia & 0.80 & 1.38 & 1.14 & 1.06 & 0.79 & 0.57 & 0.31 & 0.16 & 0.32 & 0.55 & 0.85 & 1.10 & 1.34 \\
Middle_East & 3.39 & 5.85 & 4.83 & 4.48 & 3.34 & 2.43 & 1.36 & 0.72 & 1.40 & 2.35 & 3.63 & 4.67 & 5.68 \\
India & 1.11 & 1.86 & 1.54 & 1.44 & 1.09 & 0.82 & 0.48 & 0.28 & 0.49 & 0.79 & 1.18 & 1.50 & 1.81 \\
Rest_South_Asia & 1.11 & 1.87 & 1.55 & 1.44 & 1.09 & 0.81 & 0.47 & 0.27 & 0.48 & 0.78 & 1.18 & 1.51 & 1.82 \\
South_Korea & 1.24 & 2.14 & 1.77 & 1.64 & 1.22 & 0.88 & 0.49 & 0.25 & 0.50 & 0.85 & 1.32 & 1.71 & 2.08 \\
North_Korea & 0.99 & 1.71 & 1.41 & 1.31 & 0.97 & 0.70 & 0.39 & 0.20 & 0.40 & 0.68 & 1.06 & 1.36 & 1.66 \\
China & 0.99 & 1.72 & 1.42 & 1.31 & 0.98 & 0.71 & 0.39 & 0.20 & 0.40 & 0.68 & 1.06 & 1.37 & 1.66 \\
Taiwan & 7.20 & 12.46 & 10.29 & 9.53 & 7.09 & 5.13 & 2.83 & 1.47 & 2.93 & 4.96 & 7.70 & 9.93 & 12.10 \\
Thailand & 1.21 & 1.86 & 1.57 & 1.50 & 1.20 & 0.97 & 0.66 & 0.48 & 0.66 & 0.92 & 1.28 & 1.56 & 1.83 \\
Rest_Southeastern_Asia & 1.02 & 1.58 & 1.33 & 1.27 & 1.01 & 0.81 & 0.55 & 0.39 & 0.55 & 0.77 & 1.08 & 1.32 & 1.56 \\
Indonesia & 1.00 & 0.77 & 0.77 & 0.94 & 1.01 & 1.15 & 1.16 & 1.15 & 1.10 & 1.05 & 1.03 & 0.94 & 0.88 \\
Japan & 0.53 & 0.92 & 0.76 & 0.70 & 0.52 & 0.38 & 0.21 & 0.11 & 0.22 & 0.37 & 0.57 & 0.73 & 0.89 \\
Australia & 0.79 & 0.17 & 0.30 & 0.57 & 0.82 & 1.12 & 1.28 & 1.36 & 1.20 & 1.01 & 0.80 & 0.55 & 0.33 \\
New_Zealand & 0.77 & 0.16 & 0.29 & 0.55 & 0.80 & 1.10 & 1.25 & 1.33 & 1.18 & 0.99 & 0.78 & 0.53 & 0.31 \\
Rest_Oceania & 1.36 & 0.53 & 0.69 & 1.07 & 1.40 & 1.82 & 2.01 & 2.10 & 1.89 & 1.64 & 1.39 & 1.05 & 0.76 \\
\hline
\end{tabular}



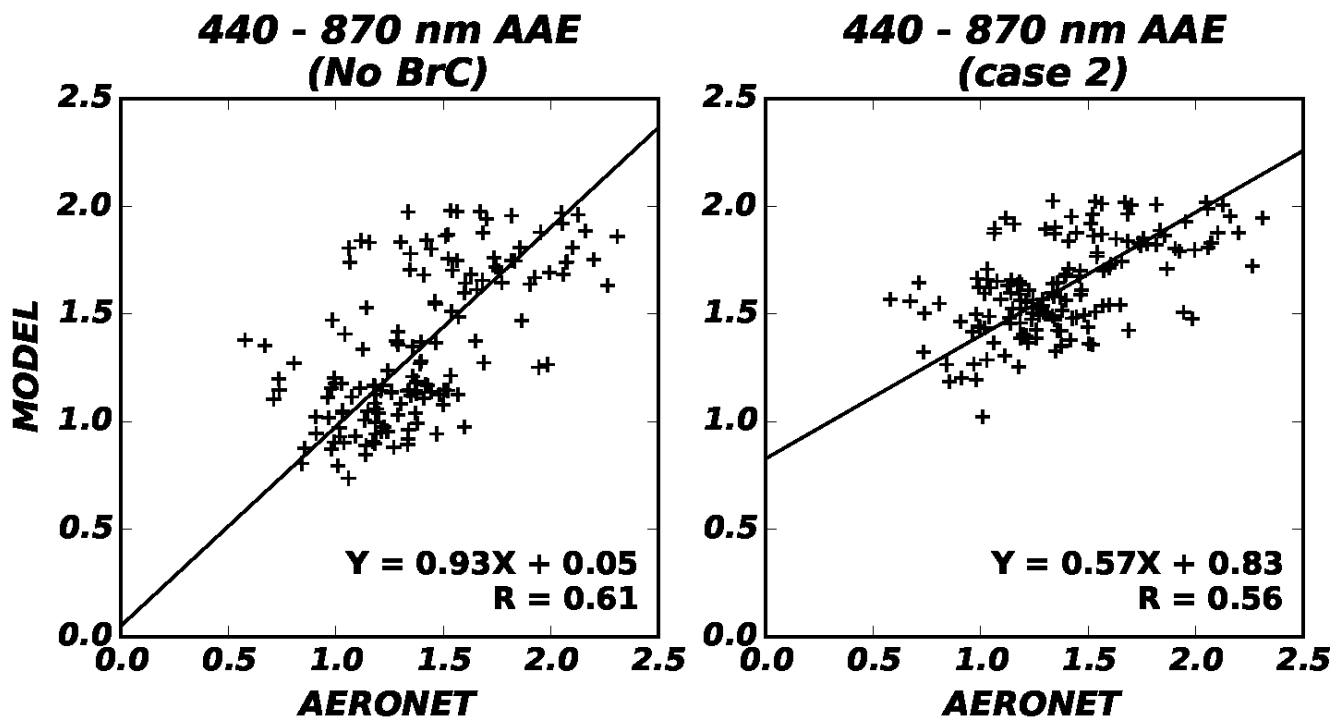

Figure S1. Scatterplots of simulated versus AERONET 440 - $870 \mathrm{~nm}$ AAE for the without BrC (left) and with BrC of case 2 (right).
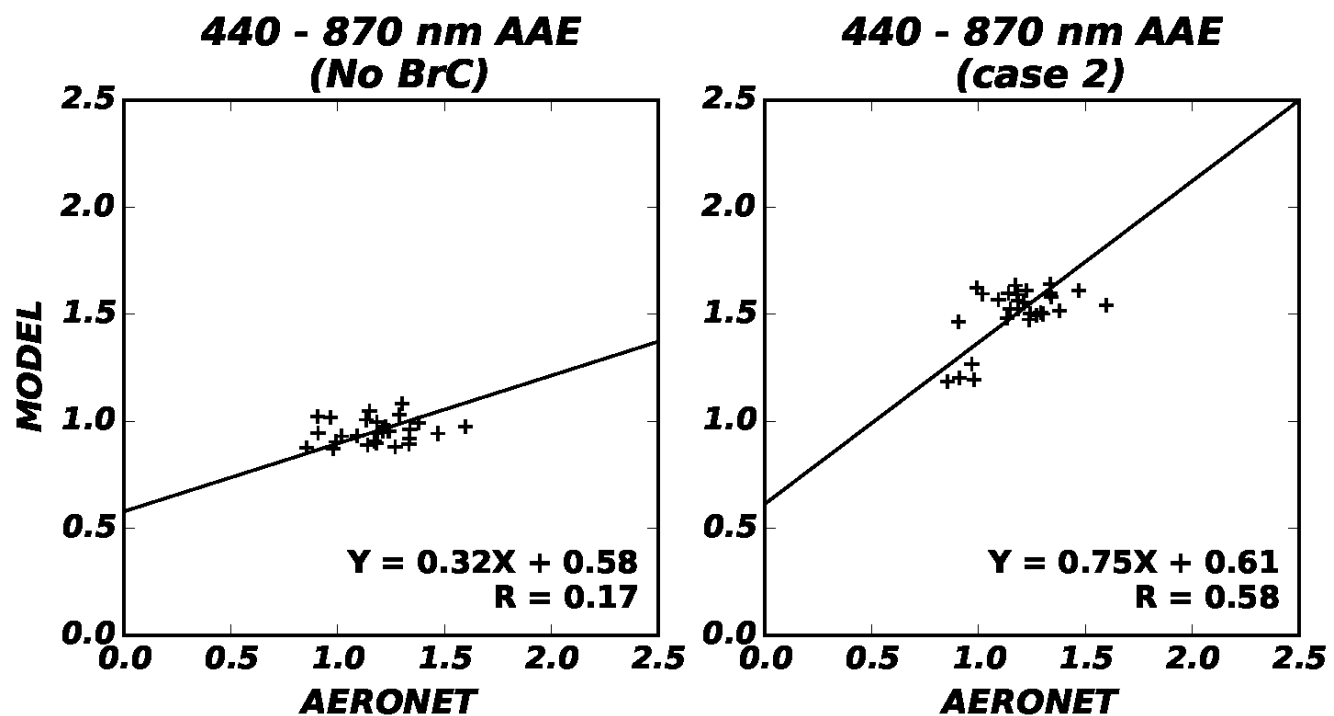

Figure S2. Same as Figure S1 above but for the regions where the $\mathrm{BC}$ emission ratios between our model and top-down estimates from Cohen and Wang (2014) are less than 2. 

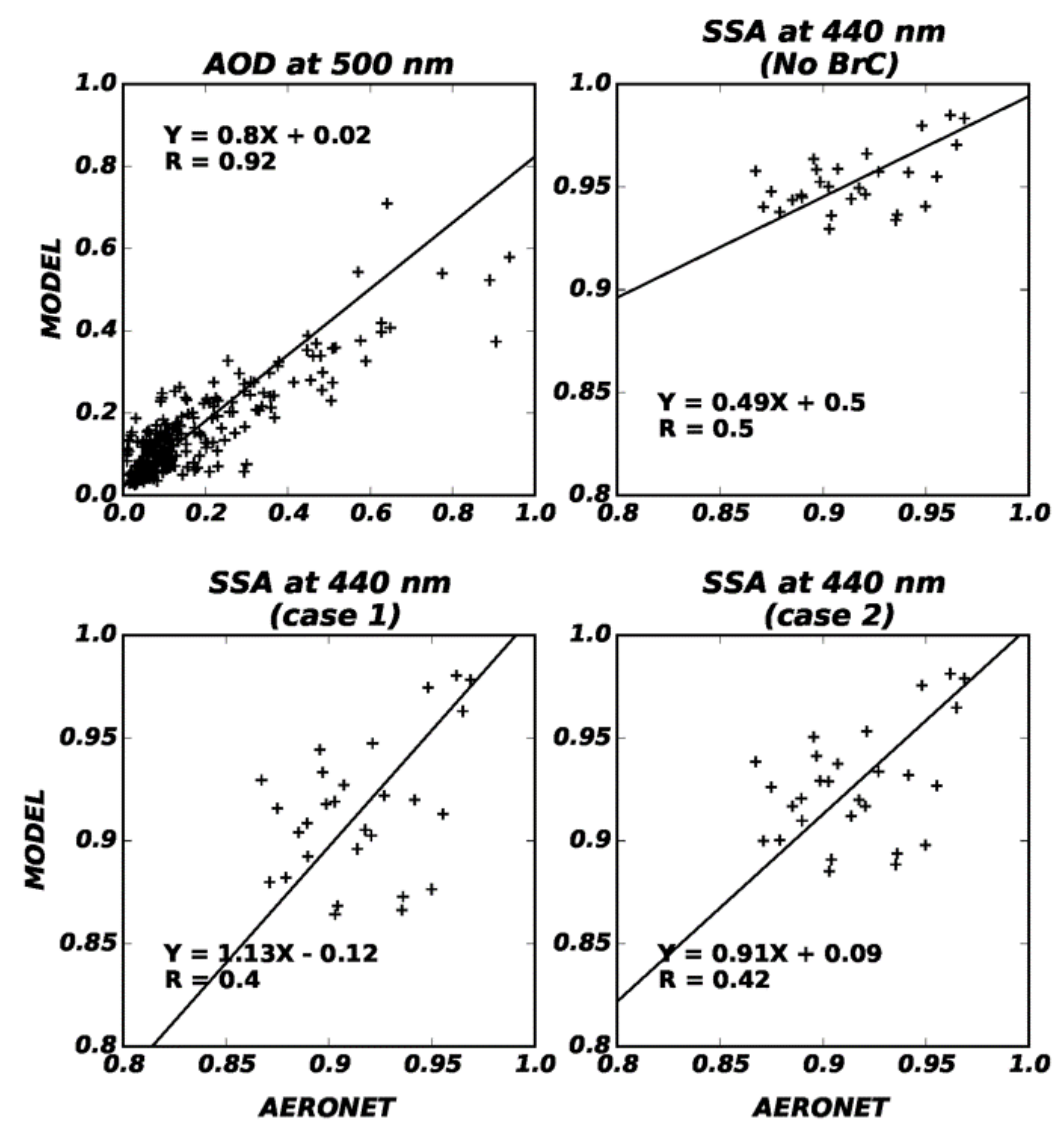

Figure S3. Same as Figure 5 but for the regions where the BC emission ratios between our model and top-down estimates from Cohen and Wang (2014) are less than 2.

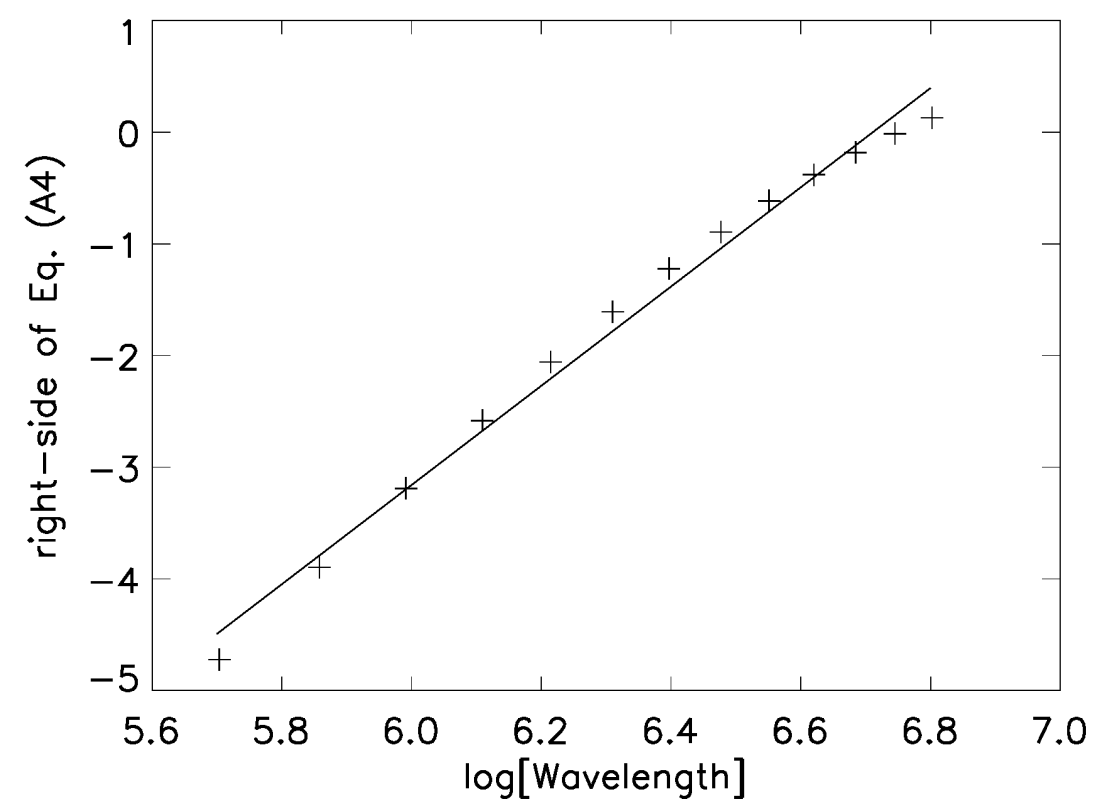

Figure S4. Linear regression fitting of Eq. (A4) when $F=4.0$. $X$-axis is logarithm of wavelength and Y-axis is right side of Eq. (A4). 